\title{
A Systematic Review of the Effect of Probiotic Supplementation on Schizophrenia Symptoms
}

\author{
Qin Xiang $\mathrm{Ng}^{\mathrm{a}, \mathrm{b}}$ Alex Yu Sen Soh ${ }^{c}$ Nandini Venkatanarayanan ${ }^{\mathrm{c}}$ \\ Collin Yih Xian $\mathrm{Ho}^{\mathrm{a}}$ Donovan Yutong Lim ${ }^{\mathrm{d}}$ Wee-Song $\mathrm{Yeo}^{\mathrm{c}}$

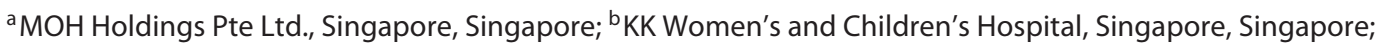 \\ 'National University Hospital, National University Health System, Singapore, Singapore; ${ }^{d}$ Department of Child and \\ Adolescent Psychiatry, Institute of Mental Health, Singapore, Singapore
}

\section{Keywords}

Schizophrenia · Probiotics · Supplementation ·

Lactobacillus · Gut-brain axis

\begin{abstract}
Background: Derangements of the gut microbiome have been linked to increased systemic inflammation and central nervous system disorders, including schizophrenia. This systematic review thus aimed to investigate the hypothesis that probiotic supplementation improves schizophrenia symptoms. Methods: By using the keywords (probiotic OR gut OR microbiota OR microbiome OR yogurt OR yoghurt OR lactobacillus OR bifidobacterium) AND (schizophrenia OR psychosis), a preliminary search of the PubMed, Medline, Embase, Google Scholar, ClinicalTrials.gov, Clinical Trials Register of the Cochrane Collaboration Depression, Anxiety and Neurosis Group (CCDANTR), and Cochrane Field for Complementary Medicine databases yielded 329 papers published in English between January 1, 1960 and May 1, 2018. Attempts were made to search grey literature as well. Results: Three clinical studies were reviewed, comparing the use of probiotics to placebo controls. Applying per-protocol analysis and a fixed-effects model, there was no significant differ-
\end{abstract}

\section{KARGER}

(c) 2019 S. Karger AG, Basel

E-Mail karger@karger.com

www.karger.com/nps ence in schizophrenia symptoms between the group that received probiotic supplementation and the placebo group post-intervention as the standardized mean difference was $-0.0884(95 \% \mathrm{Cl}-0.380$ to $0.204, p=0.551)$. Separate analyses were performed to investigate the effect of probiotic supplementation on positive or negative symptoms of schizophrenia alone. In both instances, no significant difference was observed as well. Conclusion: Based on current evidence, limited inferences can be made regarding the efficacy of probiotics in schizophrenia. Although probiotics may have other benefits, for example to regulate bowel movement and ameliorate the metabolic effects of antipsychotic medications, the clinical utility of probiotics in the treatment of schizophrenia patients remains to be validated by future clinical studies.

(c) 2019 S. Karger AG, Basel

\section{Introduction}

Although schizophrenia affects less than 1\% of the global population [1], it is a debilitating chronic condition that carries significant physical and mental morbidity and socioeconomic costs [2]. In the USA, the total di- 
rect and indirect costs of schizophrenia in 2013 was estimated to be a staggering USD 155.7 billion [3]. Patients with schizophrenia suffer from a combination of "positive" psychotic symptoms (e.g., delusions and hallucinations) and "negative" symptoms (e.g., blunted affect, social withdrawal, and avolition of speech) [4]. Negative symptoms are particularly difficult to treat, and current psychopharmacological options remain limited [5]. The persistence of these negative symptoms results in the loss of cognitive functions and productivity, inevitably contributing to the immense personal and socioeconomic costs of schizophrenia.

Increasing research into the pathogenesis of schizophrenia has implicated the potential role of the human microbiome and "gut-brain axis," i.e., the bidirectional signaling between the central and enteric nervous system [6]. Deranged gut microbiota has been associated with increased systemic inflammation [7], and the resulting neuroinflammation is thought to be directly linked to schizophrenia [8]. In a recent study, patients with firstepisode psychosis were found to have a markedly altered fecal microbiota taxonomic signature compared to nonpsychiatric controls, with a relatively increased abundance of Lactobacillaceae, Halothiobacillaceae, Brucellaceae, and Micrococcineae, and decreased Veillonellaceae [9]. This was particularly evident in schizophrenic patients with more negative symptoms and poorer global functioning.

By extension, probiotic supplementation could thus be potentially beneficial for tackling the negative symptoms in patients with schizophrenia. Probiotic supplementation has been shown to have sustained effects on gut microbiota [10] and probiotics have demonstrated anti-inflammatory and immune-modulatory properties [11]. Like several natural products and nutraceuticals [12], probiotics have gained enormous popularity for myriad clinical applications, including gastrointestinal disorders and mood disorders [13, 14]. As no previous systematic review or meta-analysis has been done to investigate the effect of probiotic supplementation on schizophrenia symptoms, this study aims to provide the first review on the topic and generate hypotheses for future research.

\section{Methods}

To achieve a high standard of reporting, we adopted the Preferred Reporting Items for Systematic Reviews and Meta-Analyses (PRISMA) guidelines [15] and the statement that consists of a 27item checklist and a 4-phase flow diagram for reporting. By using the keywords (probiotic OR gut OR microbiota OR microbiome OR yogurt OR yoghurt OR lactobacillus OR bifidobacterium) AND (schizophrenia OR psychosis), a preliminary search of the PubMed, Medline, Embase, Google Scholar, ClinicalTrials.gov, Clinical Trials Register of the Cochrane Collaboration Depression, Anxiety and Neurosis Group (CCDANTR), and Cochrane Field for Complementary Medicine databases yielded 329 papers published in English between January 1, 1960 and May 1, 2018. Grey literature was searched for using the Google search engine and the Open System for Information on Grey Literature in Europe (OpenSIGLE) database. The study investigators (Q.X.N., N.V., and C.Y.X.H.) performed independent title/abstract screening in order to identify articles of interest. For relevant abstracts, full articles were obtained, reviewed, and also checked for references of interest. If necessary, the authors of the articles were contacted to provide additional data. The review protocol was not prospectively registered.

Full articles were reviewed by 3 investigators (Q.X.N., N.V., and C.Y.X.H.) for inclusion. Any disagreement was resolved by discussion and consensus amongst the 3 investigators. The inclusion criteria for this review were as follow: (1) a published clinical trial, (2) specified dose of probiotic administered as an active intervention, and (3) schizophrenia symptoms were quantified using a validated rating scale, e.g., Positive and Negative Syndrome Scale (PANSS) pre- and post-intervention.

The methodological quality of the eligible clinical trials was assessed using the Cochrane Collaboration's tool for assessing risk of bias [16] by discussion and consensus amongst all study investigators. Information on the study design, study population, and outcome measures of the studies reviewed were extracted and summarized in Table 1 . The primary outcome measure of interest was Cohen's d, the calculated standardized mean difference (SMD) for change in psychotic symptoms, as quantified using a validated rating scale, e.g., PANSS from baseline with intervention. Estimates were pooled and the corresponding 95\% confidence intervals ( $95 \% \mathrm{CI}$ ) and $p$ values were calculated. Heterogeneity amongst the different studies pooled was assessed using the $I^{2}$ statistic and Cochran's Q test. If heterogeneity was small $\left(I^{2} \leq 50 \%\right)$, a fixedeffects model was applied for the meta-analysis. All statistical analyses were performed using MedCalc Statistical Software version 14.8.1 (MedCalc Software bvba, Ostend, Belgium; http://www. medcalc.org; 2014).

\section{Results}

The literature search and abstraction process is summarized in Figure 1. No studies were found through the grey literature search. Table 1 shows the salient details of the three clinical studies reviewed. None of the authors had to be contacted to provide additional data. As the number of available studies were small $(<10)$, a funnel plot or Egger test was not performed to assess publication bias and sensitivity analysis was not done. In fact, all three published studies were based on the same clinical trial (ClinicalTrials.gov identifier NCT01242371). 
Table 1. Characteristics of the studies included in this review

\begin{tabular}{|c|c|c|c|c|c|c|c|}
\hline $\begin{array}{l}\text { First author, } \\
\text { year }\end{array}$ & Study design & Study sample & $\begin{array}{l}\text { Probiotic dosage and } \\
\text { duration }\end{array}$ & $\begin{array}{l}\text { Diagnosis } \\
\text { criteria }\end{array}$ & $\begin{array}{l}\text { Outcome } \\
\text { measures }\end{array}$ & $\begin{array}{l}\text { Country } \\
\text { of origin }\end{array}$ & Conclusions \\
\hline $\begin{array}{l}\text { Dickerson } \\
{[17], 2014}\end{array}$ & $\begin{array}{l}\text { Randomized, } \\
\text { placebo-con- } \\
\text { trolled, double- } \\
\text { blind trial }\end{array}$ & $\begin{array}{l}n=58 \text {, patients with at } \\
\text { least moderately } \\
\text { severe psychotic } \\
\text { symptoms, aged } 18- \\
65 \text { years }\end{array}$ & $\begin{array}{l}\text { Combined L. rhamnosus } \\
\text { strain GG and B. animalis } \\
\text { subsp. lactis strain Bb12 for } \\
14 \text { weeks }\end{array}$ & DSM-IV & PANSS & USA & $\begin{array}{l}\text { Probiotic supplemen- } \\
\text { tation had no signifi- } \\
\text { cant difference on } \\
\text { PANSS scores }\end{array}$ \\
\hline
\end{tabular}

DSM-IV, Diagnostic and Statistical Manual of Mental Disorders, 4th edition; PANSS, Positive and Negative Syndrome Scale

It was encouraging that these [17-19] were randomized, placebo-controlled, double-blind studies with a generally low risk of bias (Table 2). However, the method for generation of a randomized sequence was unclear in all of the studies. The latter studies $[18,19]$ were also follow-up studies conducted using the same patient population [17]. None of the three studies found a significant difference for probiotic supplementation on PANSS scores.

\section{Symptoms, Comparing Probiotic Supplementation, and Placebo}

In all three studies, patients were maintained on their regular antipsychotic treatment, with or without adjunctive probiotic supplementation. As seen in Figure 2, applying per-protocol analysis and a fixed-effects model, there was no statistically significant difference in schizophrenia symptoms (total PANSS score) between the group that received probiotic supplementation and the placebo group post-intervention as the SMD (Cohen's d) was -0.0884 (95\% CI -0.380 to 0.204 , $p=0.551)$. A fixed-effects model was chosen as all three studies were drawn from the same patient population. Separate analyses were performed to investigate the effect of probiotic supplementation on "positive" psychotic symptoms (e.g., delusions and hallucinations) or

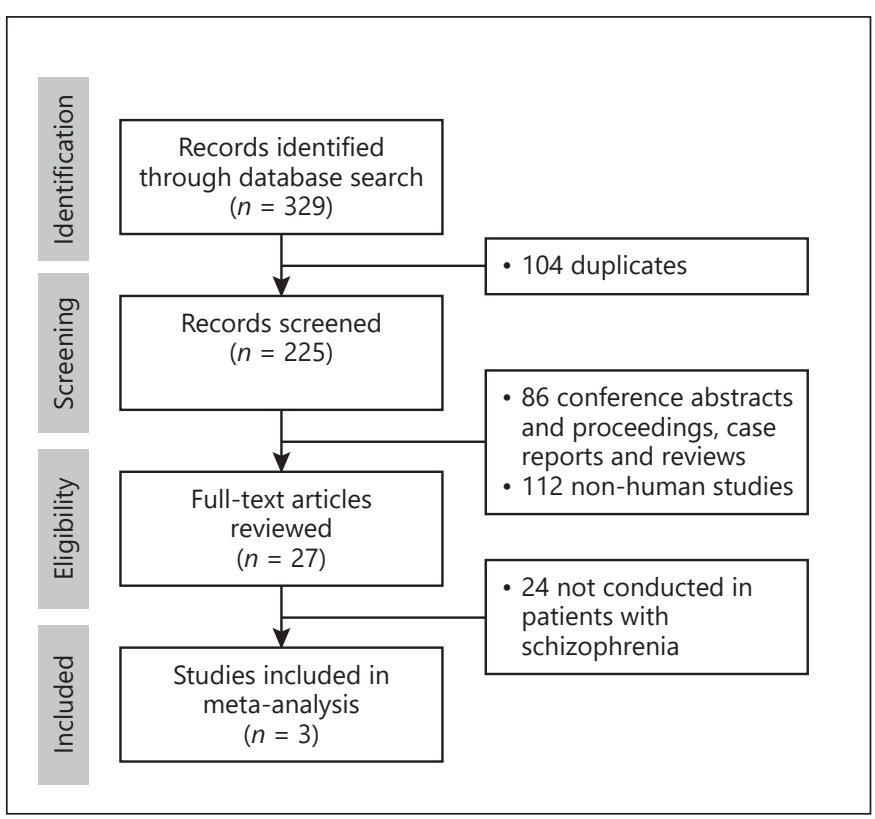

Fig. 1. The literature search and abstraction process.

"negative" symptoms (e.g., blunted affect, social withdrawal, and avolition of speech) alone. In both instances, no significant difference was observed as well. 
Table 2. Results of the Cochrane Collaboration's tool for assessing risk of bias

\begin{tabular}{|c|c|c|c|c|c|c|}
\hline Study & $\begin{array}{l}\text { Sequence } \\
\text { generation }\end{array}$ & $\begin{array}{l}\text { Allocation } \\
\text { concealment }\end{array}$ & Blinding & $\begin{array}{l}\text { Incomplete } \\
\text { outcome data }\end{array}$ & $\begin{array}{l}\text { Selective outcome } \\
\text { reporting }\end{array}$ & $\begin{array}{l}\text { Other } \\
\text { bias }\end{array}$ \\
\hline Dickerson [16], 2014 & $?$ & + & + & + & + & $?$ \\
\hline Severance [17], 2017 & $?$ & + & + & + & + & $?$ \\
\hline Tomasik [18], 2015 & $?$ & + & + & + & + & $?$ \\
\hline
\end{tabular}

+ , low risk of bias; ?, unclear risk of bias.

\section{Discussion}

This is the first systematic review reporting the effect of probiotic supplementation on schizophrenia symptoms. Despite promising preclinical data on the anti-inflammatory and immunomodulatory effects of probiotics [11] and the hypothesized link between immune dysfunction, neuroinflammation, and schizophrenia pathogenesis, there is a dearth of clinical studies on the effects of probiotic supplementation on schizophrenia symptoms. Only one clinical trial was identified despite a thorough and systematic literature search. All three published studies were based on the same clinical trial (ClinicalTrials. gov identifier NCT01242371). Current evidence does not support an adjunctive role of probiotics in the management of schizophrenia, as the effects on schizophrenia symptoms were statistically insignificant (SMD -0.0884 , $95 \%$ CI -0.380 to $0.204, p=0.551$ ).

Although no significant improvements in schizophrenia symptoms were observed with probiotic supplementation, other potential functions may exist, especially since probiotics are generally safe and well tolerated [20]. There is evidence that probiotics may alleviate the unwanted constipation [17, 21], weight gain, and adverse metabolic effects [22] commonly associated with antipsychotic use. In the study by Tomasik et al. [19], probiotic supplementation was found to significantly increase brain-derived neurotrophic factor (BDNF) levels. BDNF has a postulated role in neuroplasticity and neuronal survival [23], and alterations in BDNF have been associated with the cognitive deficits common in chronic schizophrenia [24].

Moreover, derangements of gut microbiota contribute to elevated serum levels of pro-inflammatory cytokines [7] and a correlation between the level of inflammatory markers and severity of psychotic symptoms has been reported [25]. At present, neuroinflammation [8] and immune dysfunction [26] have been implicated in the pathogenesis of schizophrenia. The human microbiome is

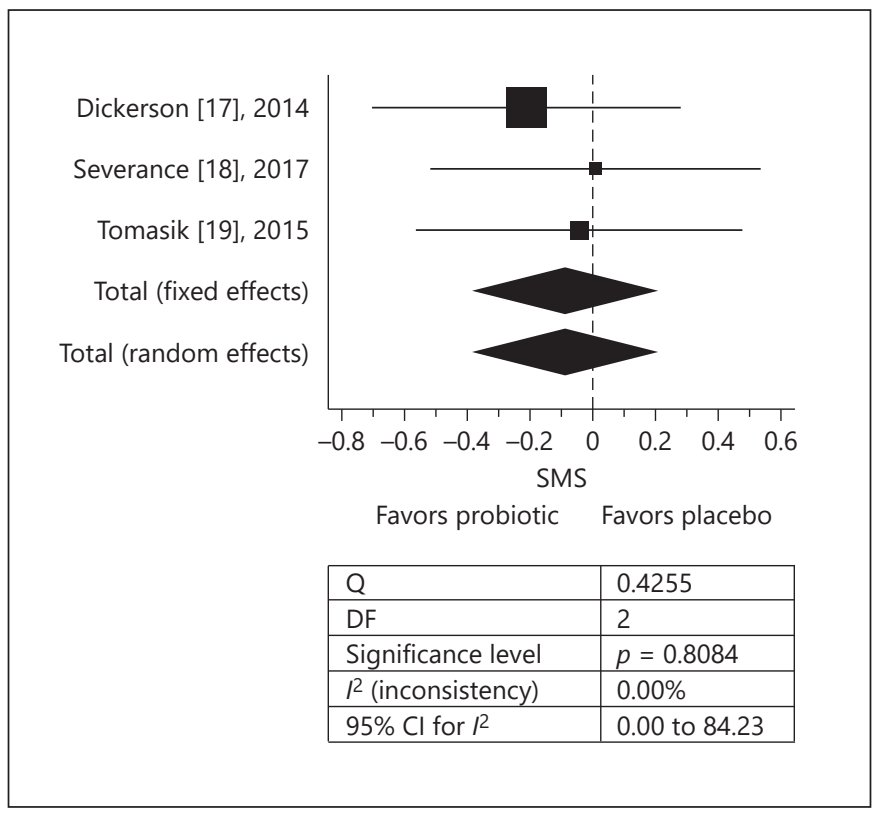

Fig. 2. Per-protocol analysis and the fixed-effects model.

thought to modulate inflammation and immune function and act either directly or indirectly through microbial metabolites. Although gaps exist in our current understanding of the interplay between gut microbiota and host immunity, gut microbes may produce bioactive "immunomodulins" and regulatory cytokines such as interleukin-10 and tumor necrosis factor- $\beta$ that attenuate systemic inflammation [27]. Furthermore, microbial biosynthesis and modulation of neurotransmitters that cross the blood-brain barrier such as serotonin [28] and $\gamma$-aminobutyric acid (GABA) [29] can influence brain function and affect.

Moving forward, there is a clear need for more randomized, controlled trials to strengthen the current evidence base. There is a paucity of clinical studies and fu- 
ture clinical trials should also report secondary outcomes such as metabolic indices, to further explore the potential benefits of probiotic supplementation. Further mechanistic studies enabled by the advent of "omics" technology would also be helpful [30]. It is also vital that future studies continue to report the specific strain of probiotics used as the efficacy of probiotics is known to be strain and disease specific [31].

\section{Conclusion}

This is the first systematic review and meta-analysis investigating the effect of probiotic supplementation on schizophrenia symptoms. At present, limited inferences can be made regarding the efficacy of probiotics in schizophrenia. Despite promising preclinical data on the antiinflammatory and immunomodulatory effects of probiotics and the hypothesized link between immune dysfunction, neuroinflammation, and schizophrenia pathogenesis, there is a paucity of clinical studies to support the benefits of probiotic supplementation in patients with schizophrenia. The pooled SMD of -0.0884 (95\% CI -0.380 to $0.204, p=0.551$ ) based on three studies indicates the non-significant effect of probiotics on schizophrenia symptoms. Although probiotics may have other benefits, e.g., to regulate bowel movement and ameliorate the metabolic effects of antipsychotic medications, the clinical use of probiotics in schizophrenia cannot be recommended until more studies are available. The clinical utility of probiotics in the treatment of schizophrenia patients remains to be validated by future clinical studies.

\section{Disclosure Statement}

The authors report no conflicts of interest. This research did not receive any specific grant from funding agencies in the public, commercial, or not-for-profit sectors. The authors alone are responsible for the content and writing of the article.

\section{References}

1 Saha S, Chant D, Welham J, McGrath J. A systematic review of the prevalence of schizophrenia. PLoS Med. 2005 May;2(5):e141.

2 Knapp M, Mangalore R, Simon J. The global costs of schizophrenia. Schizophr Bull. 2004; 30(2):279-93.

3 Cloutier M, Aigbogun MS, Guerin A, Nitulescu R, Ramanakumar AV, Kamat SA, et al. The economic burden of schizophrenia in the United States in 2013. J Clin Psychiatry. 2016 Jun;77(6):764-71.

4 Strauss JS, Carpenter WT, Bartko JJ. Speculations on the processes that underlie schizophrenic symptoms and signs: III. Schizophr Bull. 1974;1(11):61-9.

5 Fusar-Poli P, Papanastasiou E, Stahl D, Rocchetti M, Carpenter W, Shergill S, et al. Treatments of negative symptoms in schizophrenia: meta-analysis of 168 randomized placebo-controlled trials. Schizophr Bull. 2015 Jul; 41(4):892-9.

6 Nemani K, Hosseini Ghomi R, McCormick B, Fan X. Schizophrenia and the gut-brain axis. Prog Neuropsychopharmacol Biol Psychiatry. 2015 Jan;56:155-60.

7 Rogers GB, Keating DJ, Young RL, Wong ML, Licinio J, Wesselingh S. From gut dysbiosis to altered brain function and mental illness: mechanisms and pathways. Mol Psychiatry. 2016 Jun;21(6):738-48.

$8 \mathrm{Na}$ KS, Jung HY, Kim YK. The role of proinflammatory cytokines in the neuroinflammation and neurogenesis of schizophrenia. Prog Neuropsychopharmacol Biol Psychiatry. 2014 Jan;48:277-86.
9 Schwarz E, Maukonen J, Hyytiäinen T, Kieseppä T, Orešič M, Sabunciyan S, et al. Analysis of microbiota in first episode psychosis identifies preliminary associations with symptom severity and treatment response. Schizophr Res. 2018 Feb;192:398-403.

10 Alander M, Satokari R, Korpela R, Saxelin M, Vilpponen-Salmela T, Mattila-Sandholm T, et al. Persistence of colonization of human colonic mucosa by a probiotic strain, Lactobacillus rhamnosus GG, after oral consumption. Appl Environ Microbiol. 1999 Jan;65(1):351-4.

11 Frei R, Akdis M, O’Mahony L. Prebiotics, probiotics, synbiotics, and the immune system: experimental data and clinical evidence. Curr Opin Gastroenterol. 2015 Mar;31(2): 153-8.

$12 \mathrm{Ng}$ QX, Koh SS, Chan HW, Ho CY. Clinical use of curcumin in depression: A meta-analysis. J Am Med Dir Assoc. 2017 Jun;18(6): 503-8.

13 Rondanelli M, Faliva MA, Perna S, Giacosa A, Peroni G, Castellazzi AM. Using probiotics in clinical practice: where are we now? A review of existing meta-analyses. Gut Microbes. 2017 Nov;8(6):521-43.

14 Ng QX, Peters C, Ho CY, Yutong DL, Yeo WS. A meta-analysis of the use of probiotics to alleviate depressive symptoms. J Affect Disord. 2018 Mar;228:13-9.

15 Moher D, Liberati A, Tetzlaff J, Altman DG; PRISMA Group. Preferred reporting items for systematic reviews and meta-analyses: the PRISMA statement. Ann Intern Med. 2009 Aug;151(4):264-9.
16 Higgins JP, Altman DG, Gøtzsche PC, Jüni P, Moher D, Oxman AD, et al.; Cochrane Bias Methods Group; Cochrane Statistical Methods Group. The Cochrane Collaboration's tool for assessing risk of bias in randomised trials. BMJ. 2011 Oct;343:d5928.

17 Dickerson FB, Stallings C, Origoni A, Katsafanas E, Savage CL, Schweinfurth LA, et al. Effect of probiotic supplementation on schizophrenia symptoms and association with gastrointestinal functioning: a randomized, placebo-controlled trial. Prim Care Companion CNS Disord. 2014; 16(1):PCC.13m01579.

18 Severance EG, Gressitt KL, Stallings CR, Katsafanas E, Schweinfurth LA, Savage CL, et al. Probiotic normalization of Candida albicans in schizophrenia: a randomized, placebocontrolled, longitudinal pilot study. Brain Behav Immun. 2017 May;62:41-5.

19 TomasikJ, Yolken RH, Bahn S, Dickerson FB. Immunomodulatory effects of probiotic supplementation in schizophrenia patients: a randomized, placebo-controlled trial. Biomark Insights. 2015 Jun;10:47-54.

20 Doron S, Snydman DR., 2015. Risk and safety of probiotics. Clin Infect Dis. 2015 May; 60(suppl 2):S129-34.

21 Soh AY, Kang JY, Siah KT, Scarpignato C, Gwee KA. Searching for a definition for pharmacologically refractory constipation: A systematic review. J Gastroenterol Hepatol. 2018 Mar;33(3):564-75. 
22 Sayon-Orea C, Martínez-González MA, RuizCanela M, Bes-Rastrollo M. Associations between Yogurt Consumption and Weight Gain and Risk of Obesity and Metabolic Syndrome: A Systematic Review. Adv Nutr. 2017 Jan; 8(1):146S-54S.

23 Mattson MP, Maudsley S, Martin B. BDNF and 5-HT: a dynamic duo in age-related neuronal plasticity and neurodegenerative disorders. Trends Neurosci. 2004 Oct;27(10):58994.

24 Durany N, Michel T, Zöchling R, Boissl KW, Cruz-Sánchez FF, Riederer P, et al. Brain-derived neurotrophic factor and neurotrophin 3 in schizophrenic psychoses. Schizophr Res. 2001 Oct;52(1-2):79-86.
25 Hope S, Ueland T, Steen NE, Dieset I, Lorentzen S, Berg AO, et al. Interleukin 1 receptor antagonist and soluble tumor necrosis factor receptor 1 are associated with general severity and psychotic symptoms in schizophrenia and bipolar disorder. Schizophr Res. 2013 Apr;145(1-3):36-42.

26 van Kesteren CF, Gremmels H, de Witte LD, Hol EM, Van Gool AR, Falkai PG, et al. Immune involvement in the pathogenesis of schizophrenia: a meta-analysis on postmortem brain studies. Transl Psychiatry. 2017 Mar;7(3):e1075.

27 Kemgang TS, Kapila S, Shanmugam VP, Kapila R. Cross-talk between probiotic lactobacilli and host immune system. J Appl Microbiol. 2014 Aug;117(2):303-19.
28 Yano JM, Yu K, Donaldson GP, Shastri GG, Ann $\mathrm{P}, \mathrm{Ma} \mathrm{L}$, et al. Indigenous bacteria from the gut microbiota regulate host serotonin biosynthesis. Cell. 2015 Apr;161(2):264-76.

29 Barrett E, Ross RP, O'Toole PW, Fitzgerald GF, Stanton C. $\gamma$-Aminobutyric acid production by culturable bacteria from the human intestine. J Appl Microbiol. 2012 Aug;113(2):411-7.

30 Du C, Zhang B, He Y, Hu C, Ng QX, Zhang H, et al. Biological effect of aqueous $\mathrm{C} 60$ aggregates on Scenedesmus obliquus revealed by transcriptomics and non-targeted metabolomics. J Hazard Mater. 2017 Feb;324(Pt B):221-9.

31 McFarland LV, Evans CT, Goldstein EJ. Strain-specificity and disease-specificity of probiotic efficacy: a systematic review and meta-analysis. Front Med. 2018 May;5:124. 